\title{
Calcium Supplements and Risk of Cardiovascular Disease: A Meta-Analysis of Clinical Trials
}

\author{
Seung-Kwon Myung ${ }^{1,2,3, *,+}{ }^{\text {, Hong-Bae Kim }}{ }^{4,+}$, Yong-Jae Lee ${ }^{5}$, , Yoon-Jung Choi ${ }^{6}{ }^{\circ}$ and Seung-Won Oh ${ }^{7}$ \\ 1 Department of Cancer Biomedical Science, National Cancer Center Graduate School of Cancer Science \\ and Policy, Goyang 10408, Korea \\ 2 Cancer Epidemiology Branch, Division of Cancer Epidemiology and Prevention, Research Institute, \\ National Cancer Center, Goyang 10408, Korea \\ 3 Department of Family Medicine and Center for Cancer Prevention and Detection, Hospital, \\ National Cancer Center, Goyang 10408, Korea \\ 4 Department of Family Medicine, MyongJi Hospital, Hanyang University College of Medicine, \\ Goyang 10475, Korea; hongbai96@mjh.or.kr \\ 5 Department of Family Medicine, College of Medicine, Yonsei University, Seoul 03722, Korea; \\ UKYJHOME@yuhs.ac \\ 6 Department of Preventive Medicine, Seoul National University College of Medicine, Seoul 03080, Korea; \\ hierica8@snu.ac.kr \\ 7 Department of Family Medicine, Healthcare System Gangnam Center, Seoul National University Hospital, \\ Seoul 06236, Korea; sw.oh@snu.ac.kr \\ * Correspondence: msk@ncc.re.kr \\ + These authors contributed equally to this work.
}

Citation: Myung, S.-K.; Kim, H.-B.; Lee, Y.-J.; Choi, Y.-J.; Oh, S.-W. Calcium Supplements and Risk of Cardiovascular Disease: A

Meta-Analysis of Clinical Trials. Nutrients 2021, 13, 368 https://doi.org/10.3390/nu13020 368

Received: 21 December 2020

Accepted: 21 January 2021

Published: 26 January 2021

Publisher's Note: MDPI stays neutral with regard to jurisdictional claims in published maps and institutional affiliations.

Copyright: (c) 2021 by the authors. Licensee MDPI, Basel, Switzerland. This article is an open access article distributed under the terms and conditions of the Creative Commons Attribution (CC BY) license (https:// creativecommons.org/licenses/by/ $4.0 /)$.

\begin{abstract}
Background: Recent systematic reviews and meta-analyses of randomized, double-blind, placebo-controlled trials (double-blind, placebo-controlled RCTs) have reported controversial findings regarding the associations between calcium supplements on the risk of cardiovascular disease (CVD). This meta-analysis aimed to investigate the association between them. Methods: We searched PubMed, EMBASE, the Cochrane Library, and the bibliographies of relevant articles for double-blind, placebo-controlled RCTs in November, 2020. Relative risks (RRs) with 95\% confidence intervals (CIs) for the risk of cardiovascular disease were calculated using a random effects model. The main outcomes were CVD, coronary heart disease (CHD), and cerebrovascular disease. Results: A total of 13 double-blind, placebo-controlled RCTs (14,692 participants in an intervention group and 14,243 participants in a control group) were included in the final analysis. Calcium supplements significantly increased the risk of CVD (RR $1.15,95 \%$ CI $1.06-1.25, \mathrm{I}^{2}=0.0 \%, n=14$ ) and CHD (RR 1.16, $95 \%$ CI 1.05-1.28, $\left.\mathrm{I}^{2}=0.0 \%, n=9\right)$ in double-blind, placebo-controlled RCTs, specifically in healthy postmenopausal women. In the subgroup meta-analysis, dietary calcium intake of 700-1000 mg per day or supplementary calcium intake of $1000 \mathrm{mg}$ per day significantly increased the risk of CVD and CHD. Conclusions: The current meta-analysis found that calcium supplements increased a risk of CVD by about $15 \%$ in healthy postmenopausal women.
\end{abstract}

Keywords: calcium supplements; cardiovascular disease; randomized controlled trials; meta-analysis

\section{Introduction}

Current guidelines for the prevention and treatment of osteoporosis recommend adequate intakes of dietary calcium ranging 700-1200 mg/day for adults aged 50 and older from health and academic organizations such as the National Osteoporosis Foundation in 2014, American Association of Clinical Endocrinologists and American College of Endocrinology in 2016, and National Osteoporosis Guideline Group in 2017 [1-3]. If dietary intakes are insufficient, calcium supplements are recommended. However, Bolland et al. raised concerns that calcium supplements were associated with an increased risk of myocardial infarction by about $30 \%$ in their meta-analysis of randomized, double-blind, 
placebo-controlled trials (double-blind, placebo-controlled RCTs) in 2010 [4] and in their updated meta-analysis published in 2011 [5]. Lewis et al. reported that there was no significant association between calcium supplementation and the risk of coronary heart disease (CHD) in the meta-analysis of randomized controlled trials [6], claiming that none of the trials included in Bolland et al.'s meta-analysis had cardiovascular disease (CVD) as its primary endpoint, and over $65 \%$ of all the heart attacks were self-reported [7]. In response to Lewis et al.'s criticism, Bolland et al. asserted that the results did not change when they adjusted for potential confounders in secondary analyses, considerable amounts of data on heart attacks were obtained from death certificates and medical records [8], and the results of Lewis et al.'s meta-analysis were similar to those from their previous meta-analysis, when an open-label study was excluded [9].

In the meantime, based on the existing peer-reviewed scientific literature, including Chung et al.'s updated systematic review and meta-analysis that there was no significant difference between calcium supplementation with or without vitamin D supplementation [10], the National Osteoporosis Foundation and the American Society for Preventive Cardiology announced a clinical guideline that there is moderate-quality evidence that calcium with or without vitamin D intake from food or supplements has no association with CVD in generally healthy adults [11].

In sum, recent systematic reviews and meta-analyses of double-blind, placebo-controlled RCTs have reported controversial findings regarding the associations between calcium supplements on the risk of CVD. Thus, we investigated the associations between the use of calcium supplements and the risk of CVD by conducting a comprehensive meta-analysis of doubleblind, placebo-controlled RCTs with various subgroup analyses according to important factors that can affect the results and also compared the differences in main findings and conclusions between the previous systematic reviews and meta-analyses and the current study.

\section{Materials and Methods}

\subsection{Data Sources and Search}

We searched PubMed, EMBASE, and the Cochrane Library using common keywords related to calcium supplements and the risk of CVD from inception to November, 2020 without language restrictions. The keywords were as follows: "calcium supplements," "calcium supplementation," and "calcium intake," for exposure factors; "cardiovascular disease," "coronary heart disease," "ischemic heart disease," "myocardial infarction," "angina," "heart failure," "cerebrovascular disease," and "stroke" for outcome factors; "randomized controlled study" for study design. We combined the above search terms with AND and OR. Calcium supplementation was defined as the use of elemental calcium supplements of at least $500 \mathrm{mg} / \mathrm{d}$, which is a conventionally used dosage, as carbonate, citrate, or gluconate. We reviewed the bibliographies of relevant articles to find out additional publications from the previous review articles and reference lists. We did not conduct a grey literature search.

\subsection{Study Selection and Eligibility}

Using the PICO (patient, problem or population; intervention; comparison, control or comparator; and outcome) criteria for eligibility criteria, we included double-blind, placebo-controlled RCTs that investigated the associations between the use of calcium supplements and the risk of cardiovascular events or mortality in adults. In this metaanalysis, we excluded open-label trials such as randomized controlled trials that did not use placebos as a control group. In the case of duplicated or shared data from the same population, we included the more comprehensive study or the most recently published one with a longer follow-up in the analysis.

Two of the authors (H.-B.K. and Y.-J.L.) independently assessed the eligibility of all studies based on the pre-determined selection criteria. Disagreements between evaluators were resolved by discussion or in consultation with a third author (S.-K.M.). 


\subsection{Assessment of the Risk of Bias}

We estimated the risk of bias based on the Cochrane Risk of Bias Tool [12] by two authors (Y.-J.C. and S.-K.M.), and those with low risk of bias ( $\geq 5$ items) were considered as an overall low risk of bias study in this analysis.

\subsection{Main and Subgroup Analysis}

For the main analysis, we investigated the associations between the use of calcium supplements and the risk of CVD, CHD, and cerebrovascular disease. CVDs are a group of disorders of the heart and blood vessels and include mainly CHD (disease of the blood vessels supplying the heart muscle, e.g., angina, myocardial infarction, and heart failure) and cerebrovascular disease (disease of the blood vessels supplying the brain, e.g., ischemic and hemorrhagic stroke). Additionally, in order to estimate the increase in absolute risk associated with calcium supplementation, we calculated the absolute risk difference by using the formula, 'absolute risk difference $=$ baseline risk (RR-1)': Baseline risk is the number of CVD or CAD events divided by the number of study participants in the placebo or control group. Subgroup meta-analyses were performed according to various factors for each disease outcome (CVD, CHD, and cerebrovascular disease) as follows: incidence or mortality, type of study population (healthy postmenopausal women and subjects with underlying disease), type of disease outcome, gender (men, women, and both), mean age of subjects ( $<55$ years and $\geq 55$ years), region (North America, Europe, and Asia), follow-up period ( $\leq 10$ years and $>10$ years), dosage of calcium supplements, and adjustment for dietary calcium intake (no and yes) for prospective cohort studies; incidence or mortality, gender (men, women, and both), mean age of subjects ( $<65$ years and $\geq 65$ years), region (Oceania, North America, and Europe), type of calcium preparation (carbonate, citrate, mixed, and not specified), duration of calcium supplementation $(<5$ years and $\geq 5$ years), dosage of calcium supplements $(500-600 \mathrm{mg} / \mathrm{d}, 1000 \mathrm{mg} / \mathrm{d}, \leq 1000 \mathrm{mg} / \mathrm{d}$, and $>1000 \mathrm{mg} / \mathrm{d}$ ), concurrent use of vitamin $\mathrm{D}$ (calcium alone and calcium plus vitamin D), number of study participants ( $\leq 1000$ and $>1000)$, daily mean dietary calcium intake $(<700 \mathrm{mg} / \mathrm{d}, 700-1000 \mathrm{mg} / \mathrm{d}$, and $>1000 \mathrm{mg} / \mathrm{d})$, and number of low risk of bias $(<5$ items and $\geq 5$ items). Additionally, in order to clarify the influence of individual trials on the summary effect estimate, a leave-one-out sensitivity meta-analysis was performed. We also compared the differences in the main findings and conclusions between the previous systematic reviews and meta-analyses and the current study.

\subsection{Statistical Analyses}

To estimate a pooled relative risk (RR) with its $95 \%$ confidence interval (CI), we calculated a pooled RR with its $95 \%$ CI by using values in cells of a $2 \times 2$ table based on an intention-to-treat analysis, whenever possible. For testing the heterogeneity across the studies, we used Higgins $\mathrm{I}^{2}$, which measures the percentage of total variation across publications [13]. $\mathrm{I}^{2}$ was computed as follows:

$$
\mathrm{I}^{2}=100 \% \times(\mathrm{Q}-\mathrm{df}) / \mathrm{Q}
$$

where $\mathrm{Q}$ is Cochran's heterogeneity statistic and $\mathrm{df}$ indicates the degrees of freedom. Negative values of $\mathrm{I}^{2}$ were set at zero; the $\mathrm{I}^{2}$ results are between $0 \%$ (no observed heterogeneity) and $100 \%$ (maximal heterogeneity). An $\mathrm{I}^{2}$ value greater than $50 \%$ was considered to indicate substantial heterogeneity [13]. A random-effects model meta-analysis on the basis of the DerSimonian and Laird method was used in the current study because individual trials were carried out in the different populations.

We estimated the publication bias using the Begg's funnel plot and Egger's test. When publication bias exists, Begg's funnel plot exhibits asymmetry or the $p$-value $<0.05$ by Egger's test. We used the Stata SE version 13.1 software package (StataCorp, College Station, TX, USA) for the statistical analysis. 


\section{Results}

\subsection{Identification of Relevant Studies}

As shown in Figure 1, out of a total of 1495 articles identified by the initial search of three databases and hand-searching relevant bibliographies, 13 double-blind, placebocontrolled RCT [14-26] were included in the final analysis.

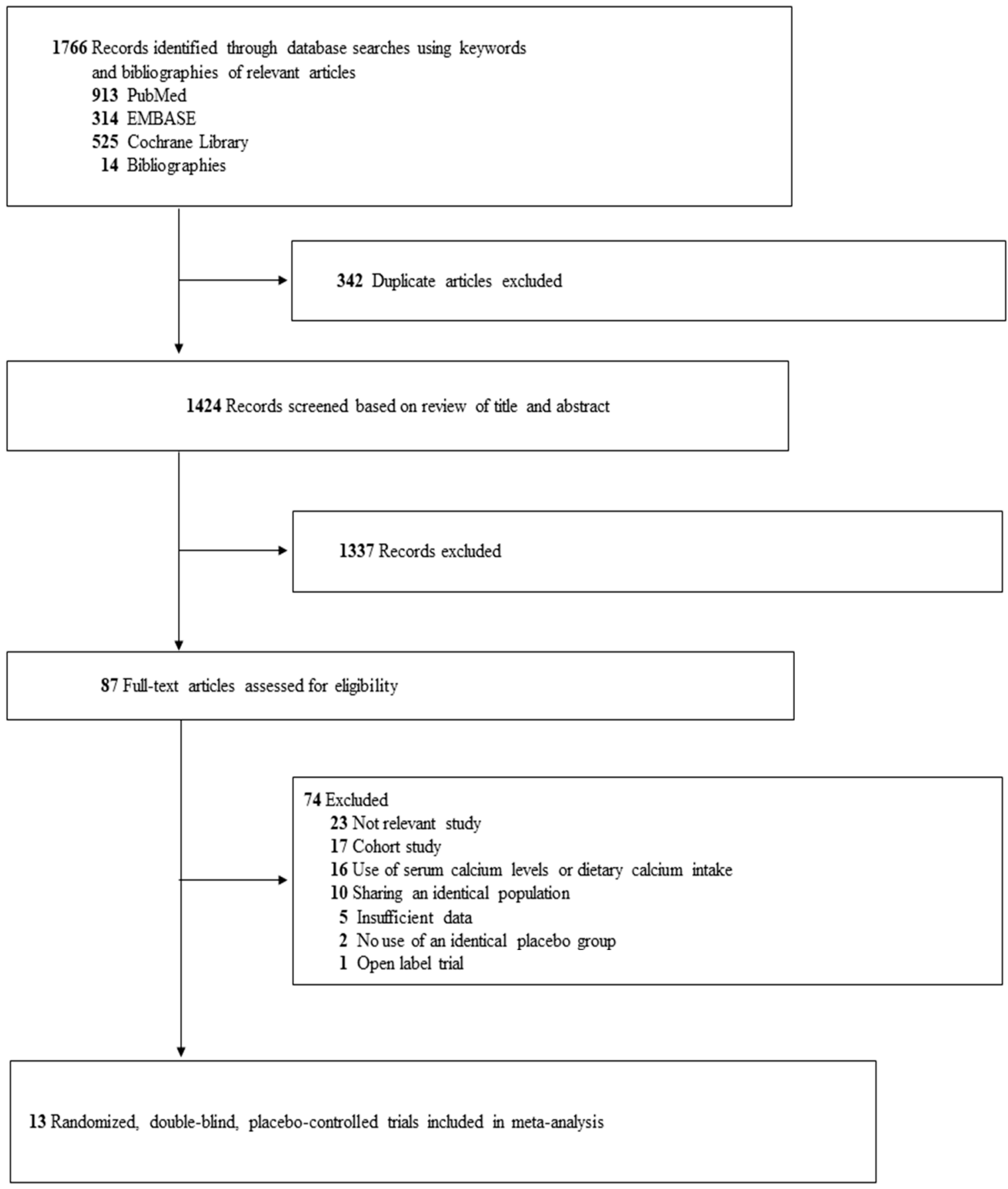

Figure 1. Study selection.

\subsection{General Characteristics of Trials}

Table 1 shows the general characteristics of 13 double-blind, placebo-controlled RCTs included in the final analysis. The included trials were published between 1990 and 2013, 
and they involved a total of 28,935 participants $(14,692$ in an intervention group and 14,243 in a control group). For trials reporting age and sex, the mean age of the study participants was 66.3 years (range, 35 to 97 years), and $92.8 \%$ of those were women. Seven trials $[16,18,19,22,24-26]$ demonstrated low risk of bias in at least five out of seven items of the Cochrane Risk of Biazs tool, while the remaining six $[14,15,17,20,21,23]$ demonstrated low risk of bias in fewer than five items (Table S1).

\subsection{Association between the Use of Calcium Supplements and Risk of CVD, CHD, and Cerebrovascular Disease}

Avenell et al.'s trial [25] used both calcium alone and calcium plus vitamin D in the supplementation groups. Thus, a total of 14 trials were included in the analysis. A random-effects meta-analysis of double-blind, placebo-controlled RCTs showed that calcium supplementation significantly increased the risks of CVD (RR 1.15, 95\% CI 1.06-1.25, $\left.\mathrm{I}^{2}=0.0 \%, n=14\right)$ and CHD (RR 1.16, 95\% CI 1.05-1.28, $\mathrm{I}^{2}=0.0 \%, n=9$ ) (Figure 2). However, the use of calcium supplements was not significantly association with the risk of cerebrovascular disease (RR 1.13, 95\% CI 0.97-1.31, $\mathrm{I}^{2}=0.0 \%, n=12$ ) (Figure 2).

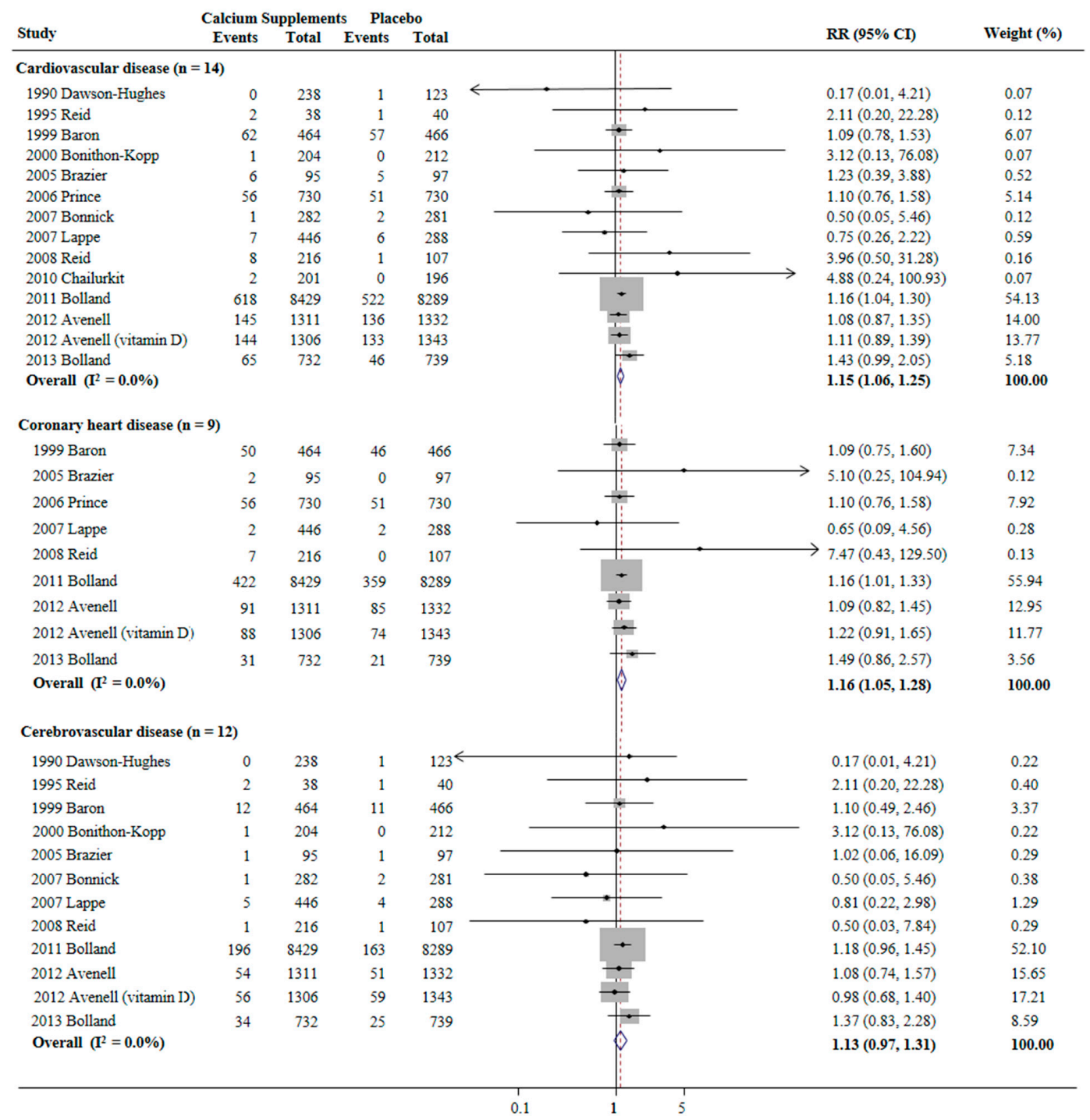

Figure 2. Use of calcium supplements and risk of cardiovascular disease in a random-effects meta-analysis of randomized controlled trials. RR, relative risk; CI, confidence interval. Avenell et al.'s trial [25] used both calcium alone and calcium plus vitamin $\mathrm{D}$ in the supplementation groups. Thus, a total of 14 trials were included in the analysis. 
Table 1. General characteristics of randomized, double-blind, placebo-controlled trials included in final analysis $(\mathrm{n}=13)$.

\begin{tabular}{|c|c|c|c|c|c|c|c|c|c|}
\hline \multirow{2}{*}{$\begin{array}{c}\text { Source } \\
\text { (Study Name) }\end{array}$} & \multirow{2}{*}{ Country } & \multirow{2}{*}{$\begin{array}{l}\text { Study Participants } \\
\text { (Mean Age, y; } \\
\text { Women, \%) }\end{array}$} & \multirow{2}{*}{$\begin{array}{c}\begin{array}{c}\text { Mean Dietary } \\
\text { Calcium Intake }\end{array} \\
(\mathrm{Mg} / \mathrm{D})\end{array}$} & \multirow{2}{*}{$\begin{array}{c}\text { Duration of } \\
\text { Supplementation, Y } \\
\text { (Follow-Up Period, Y) }\end{array}$} & \multirow{2}{*}{$\begin{array}{l}\text { Intervention } \\
\text { (vs. Placebo) }\end{array}$} & \multirow{2}{*}{ Outcomes Used } & \multicolumn{2}{|c|}{$\begin{array}{l}\text { No. of Major Cardiovascular } \\
\text { Events/No. of Participants }\end{array}$} & \multirow{2}{*}{ Data Source } \\
\hline & & & & & & & $\begin{array}{c}\text { Supplement } \\
\text { Group }\end{array}$ & $\begin{array}{l}\text { Placebo } \\
\text { Group }\end{array}$ & \\
\hline $\begin{array}{c}\text { Dawson- } \\
\text { Hughes et al., } \\
1990 \text { [14] }\end{array}$ & $\begin{array}{l}\text { United } \\
\text { States }\end{array}$ & $\begin{array}{c}361 \text { healthy } \\
\text { postmenopausal } \\
\text { women }(58.4 ; 100)\end{array}$ & 406 & $2(2)$ & $\begin{array}{c}\text { Elemental } \\
\text { calcium } 500 \\
\mathrm{mg} / \mathrm{d} \text { (as citrate } \\
\text { or citrate } \\
\text { malate) }\end{array}$ & $\begin{array}{l}\text { Stroke incidence: } \\
\text { secondary } \\
\text { endpoint }\end{array}$ & $0 / 238$ & $1 / 123$ & $\begin{array}{l}\text { Unpublished } \\
\text { data * }\end{array}$ \\
\hline $\begin{array}{l}\text { Reid et al., } \\
1995 *[15]\end{array}$ & $\begin{array}{c}\text { New } \\
\text { Zealand }\end{array}$ & $\begin{array}{c}78 \text { healthy } \\
\text { postmenopausal } \\
\text { women }(58.5 ; 100)\end{array}$ & 750 & $2(4)$ & $\begin{array}{c}\text { Elemental } \\
\text { calcium } 1000 \\
\text { mg/d (as } \\
\text { lactate- } \\
\text { gluconate } \\
\text { carbonate) }\end{array}$ & $\begin{array}{l}\text { Stroke incidence: } \\
\text { secondary } \\
\text { endpoint }\end{array}$ & $2 / 38$ & $1 / 40$ & $\begin{array}{l}\text { Unpublished } \\
\text { data * }\end{array}$ \\
\hline $\begin{array}{l}\text { Baron et al., } \\
1999 \text { [16] } \\
\text { (CPPS) }\end{array}$ & $\begin{array}{l}\text { United } \\
\text { States }\end{array}$ & $\begin{array}{l}930 \text { patients with } \\
\text { colorectal adenoma } \\
(61 ; 27.7)\end{array}$ & 880 & $4(4)$ & $\begin{array}{l}\text { Elemental } \\
\text { calcium } 1200 \\
\text { mg/d (as } \\
\text { carbonate) }\end{array}$ & $\begin{array}{l}\text { Hospitalization } \\
\text { due to cardiac } \\
\text { disease or stroke } \\
\text { incidence: } \\
\text { secondary } \\
\text { endpoint }\end{array}$ & $62 / 464$ & $57 / 466$ & $\begin{array}{l}\text { Published } \\
\text { data }\end{array}$ \\
\hline $\begin{array}{l}\text { Bonithon- } \\
\text { Kopp et al., } \\
2000 \text { [17] } \\
\text { (ECPIS) }\end{array}$ & $10 \underset{* *}{\operatorname{countries}}$ & $\begin{array}{l}640 \text { patients with a } \\
\text { history of colorectal } \\
\text { adenoma }(59.1 ; 36.4)\end{array}$ & 980 & $3(3)$ & $\begin{array}{l}\text { Elemental } \\
\text { calcium } 2000 \\
\mathrm{mg} / \mathrm{d} \text { (as } \\
\text { carbonate or } \\
\text { gluconate) }\end{array}$ & $\begin{array}{l}\text { Stroke incidence: } \\
\text { secondary } \\
\text { endpoint }\end{array}$ & $1 / 204$ & $0 / 212$ & $\begin{array}{c}\text { Unpublished } \\
\text { data * }\end{array}$ \\
\hline $\begin{array}{l}\text { Brazier et al., } \\
2005 \text { [18] }\end{array}$ & France & $\begin{array}{l}192 \text { women with } \\
\text { vitamin D deficiency } \\
(74.6 ; 100)\end{array}$ & 736 & $1(1)$ & $\begin{array}{c}\text { Elemental } \\
\text { calcium } 1000 \\
\mathrm{mg} / \mathrm{d} \text { (as } \\
\text { carbonate) }+ \\
\text { vitamin D3 } 800 \\
\text { IU /d }\end{array}$ & $\begin{array}{c}\text { Cardiovascular } \\
\text { events } \\
\text { incidence: } \\
\text { secondary } \\
\text { endpoint }\end{array}$ & $6 / 95$ & $5 / 97$ & $\begin{array}{l}\text { Published } \\
\text { data }\end{array}$ \\
\hline
\end{tabular}


Table 1. Cont.

\begin{tabular}{|c|c|c|c|c|c|c|c|c|c|}
\hline \multirow{2}{*}{$\begin{array}{c}\text { Source } \\
\text { (Study Name) }\end{array}$} & \multirow{2}{*}{ Country } & \multirow{2}{*}{$\begin{array}{l}\text { Study Participants } \\
\text { (Mean Age, y; } \\
\text { Women, \%) }\end{array}$} & \multirow{2}{*}{$\begin{array}{c}\text { Mean Dietary } \\
\text { Calcium Intake } \\
(\mathrm{Mg} / \mathrm{D})\end{array}$} & \multirow{2}{*}{$\begin{array}{c}\text { Duration of } \\
\text { Supplementation, Y } \\
\text { (Follow-Up Period, Y) }\end{array}$} & \multirow{2}{*}{$\begin{array}{l}\text { Intervention } \\
\text { (vs. Placebo) }\end{array}$} & \multirow{2}{*}{ Outcomes Used } & \multicolumn{2}{|c|}{$\begin{array}{l}\text { No. of Major Cardiovascular } \\
\text { Events/No. of Participants }\end{array}$} & \multirow{2}{*}{ Data Source } \\
\hline & & & & & & & $\begin{array}{l}\text { Supplement } \\
\text { Group }\end{array}$ & $\begin{array}{l}\text { Placebo } \\
\text { Group }\end{array}$ & \\
\hline $\begin{array}{l}\text { Bonnick et al., } \\
2007 \text { [20] }\end{array}$ & $\begin{array}{l}\text { United } \\
\text { States }\end{array}$ & $\begin{array}{l}710 \text { postmenopausal } \\
\text { women with low } \\
\text { bone mineral density } \\
(66.2 ; 100)\end{array}$ & 1240 & $2(2)$ & $\begin{array}{c}\text { Elemental } \\
\text { calcium } 1000 \\
\text { mg/d (as } \\
\text { carbonate) plus } \\
\text { alendronate } 10 \\
\text { mg/d vs. } \\
\text { alendronate } 10 \\
\text { mg/d }\end{array}$ & $\begin{array}{l}\text { Stroke incidence: } \\
\text { secondary } \\
\text { endpoint }\end{array}$ & $1 / 282$ & $2 / 281$ & $\begin{array}{l}\text { Unpublished } \\
\text { data * }\end{array}$ \\
\hline $\begin{array}{l}\text { Lappe et al., } \\
2007 *[21]\end{array}$ & $\begin{array}{l}\text { United } \\
\text { States }\end{array}$ & $\begin{array}{c}734 \text { healthy } \\
\text { postmenopausal } \\
\text { women }(66.7 ; 100)\end{array}$ & 1070 & $4(4)$ & $\begin{array}{l}\text { Elemental } \\
\text { calcium } 1500 \\
\mathrm{mg} / \mathrm{d} \text { (as } \\
\text { carbonate) or } \\
1400 \mathrm{mg} / \mathrm{d} \text { (as } \\
\text { citrate) }\end{array}$ & $\begin{array}{l}\text { MI and stroke: } \\
\text { secondary } \\
\text { endpoint }\end{array}$ & $7 / 446$ & $6 / 288$ & $\begin{array}{l}\text { Unpublished } \\
\text { data * }\end{array}$ \\
\hline $\begin{array}{l}\text { Reid et al., } \\
2008 \text { [22] }\end{array}$ & $\begin{array}{l}\text { New } \\
\text { Zealand }\end{array}$ & $\begin{array}{l}323 \text { healthy men } \\
\qquad(57 ; 0)\end{array}$ & 870 & $2(2)$ & $\begin{array}{c}\text { Elemental } \\
\text { calcium } 600 \\
\mathrm{mg} / \mathrm{d} \text { or } 1200 \\
\mathrm{mg} / \mathrm{d}\end{array}$ & $\begin{array}{l}\text { CVD events or } \\
\text { TIA: secondary } \\
\text { endpoint }\end{array}$ & $8 / 216$ & $1 / 107$ & $\begin{array}{l}\text { Published } \\
\text { data }\end{array}$ \\
\hline $\begin{array}{l}\text { Chailurkit } \\
\text { et al., } 2010 \text { [23] }\end{array}$ & Thailand & $\begin{array}{c}336 \text { physically active } \\
\text { healthy } \\
\text { postmenopausal } \\
\text { women }(65.8 ; 100)\end{array}$ & 375 & $2(2)$ & $\begin{array}{l}\text { Elemental } \\
\text { calcium } 500 \\
\text { mg/d (as } \\
\text { carbonate) }\end{array}$ & $\begin{array}{l}\text { CVD incidence: } \\
\text { secondary } \\
\text { endpoint }\end{array}$ & $2 / 201$ & 0/196 & $\begin{array}{l}\text { Published } \\
\text { data }\end{array}$ \\
\hline $\begin{array}{l}\text { Bolland et al., } \\
2011[24] \\
\text { (WHI) }\end{array}$ & $\begin{array}{l}\text { United } \\
\text { States }\end{array}$ & $\begin{array}{c}16,718 \\
\text { postmenopausal } \\
\text { women }(62.9 ; 100) \\
\text { with no personal use } \\
\text { of calcium: Reanalysis } \\
\text { of the WHI }\end{array}$ & 801 & $7(7)$ & $\begin{array}{l}\text { Calcium } 1000 \\
\text { mg/d + vitamin } \\
\text { D3 } 400 \mathrm{IU} / \mathrm{d}\end{array}$ & $\begin{array}{l}\text { Clinical MI or } \\
\text { revasculariza- } \\
\text { tion + stroke: } \\
\text { secondary } \\
\text { endpoint }\end{array}$ & $618 / 8429$ & $522 / 8289$ & $\begin{array}{l}\text { Published } \\
\text { data }\end{array}$ \\
\hline
\end{tabular}


Table 1. Cont.

\begin{tabular}{|c|c|c|c|c|c|c|c|c|c|}
\hline \multirow{2}{*}{$\begin{array}{c}\text { Source } \\
\text { (Study Name) }\end{array}$} & \multirow{2}{*}{ Country } & \multirow{2}{*}{$\begin{array}{l}\text { Study Participants } \\
\text { (Mean Age, y; } \\
\text { Women, \%) }\end{array}$} & \multirow{2}{*}{$\begin{array}{c}\begin{array}{c}\text { Mean Dietary } \\
\text { Calcium Intake }\end{array} \\
(\mathrm{Mg} / \mathrm{D})\end{array}$} & \multirow{2}{*}{$\begin{array}{c}\text { Duration of } \\
\text { Supplementation, Y } \\
\text { (Follow-Up Period, Y) }\end{array}$} & \multirow{2}{*}{$\begin{array}{l}\text { Intervention } \\
\text { (vs. Placebo) }\end{array}$} & \multirow{2}{*}{ Outcomes Used } & \multicolumn{2}{|c|}{$\begin{array}{l}\text { No. of Major Cardiovascular } \\
\text { Events/No. of Participants }\end{array}$} & \multirow{2}{*}{ Data Source } \\
\hline & & & & & & & $\begin{array}{c}\text { Supplement } \\
\text { Group }\end{array}$ & $\begin{array}{l}\text { Placebo } \\
\text { Group }\end{array}$ & \\
\hline $\begin{array}{l}\text { Bolland et al., } \\
2013 \text { [26] } \\
\text { (ACS) }\end{array}$ & $\begin{array}{l}\text { New } \\
\text { Zealand }\end{array}$ & $\begin{array}{c}1471 \text { healthy } \\
\text { postmenopausal } \\
\text { women }(74 ; 100)\end{array}$ & 857 & $5(5)$ & $\begin{array}{c}\text { Elemental } \\
\text { calcium } 1000 \\
\mathrm{mg} / \mathrm{d} \text { (as } \\
\text { citrate) }\end{array}$ & $\begin{array}{l}\text { MI or stroke: } \\
\text { secondary } \\
\text { endpoint }\end{array}$ & $65 / 732$ & $46 / 739$ & $\begin{array}{l}\text { Published } \\
\text { data }\end{array}$ \\
\hline
\end{tabular}

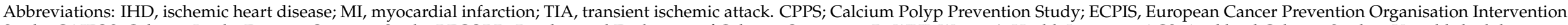

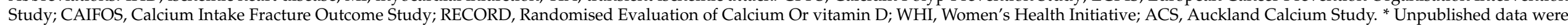
provided by authors, which were obtained from Bolland et al.'s meta-analysis article [4]. ** Belgium, Denmark, France, Germany, Ireland, Israel, Italy, Portugal, Spain, and United Kingdom. 
Publication bias was not observed: the Begg's funnel plots were all symmetrical, and $p$ for bias was 0.81 for CVD, 0.81 for CHD, and 0.32 for cerebrovascular disease in the Egger's test (Figure 3).

A. Cardiovascular Disease

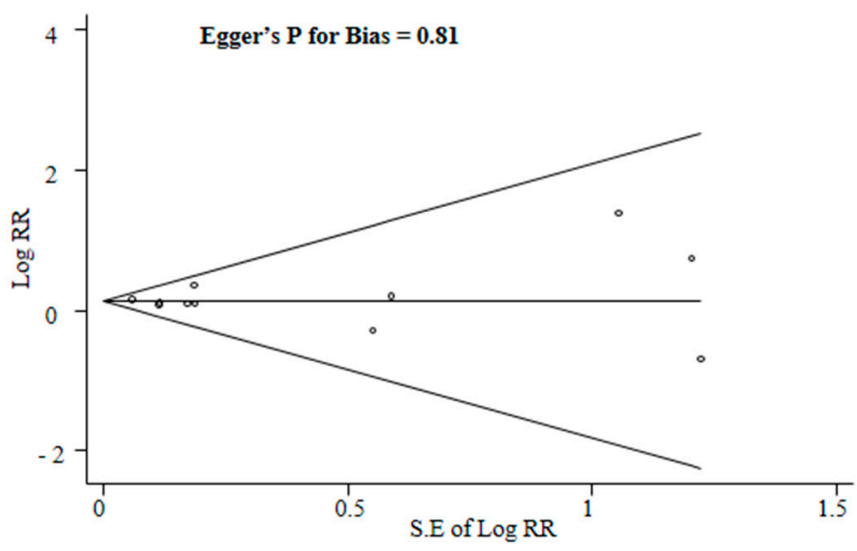

B. Coronary Heart Disease

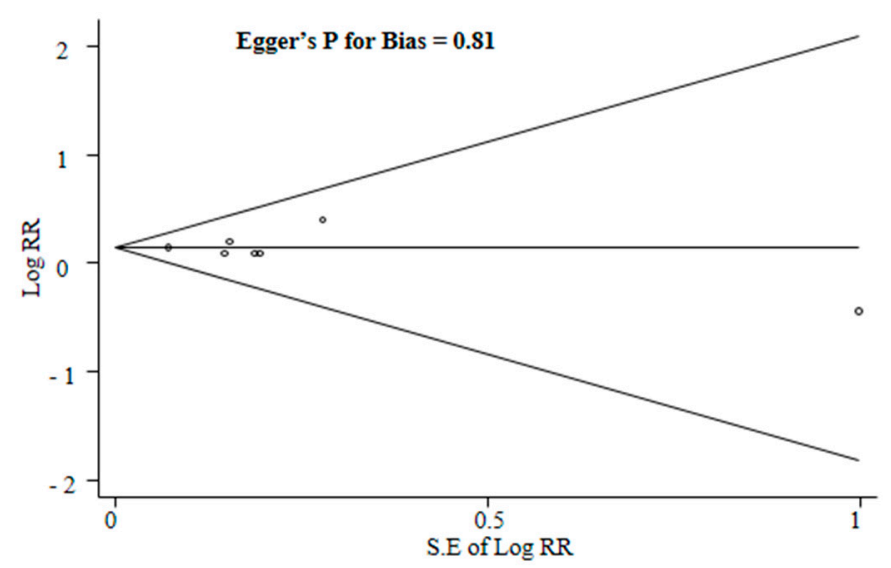

C. Cerebrovascular Disease

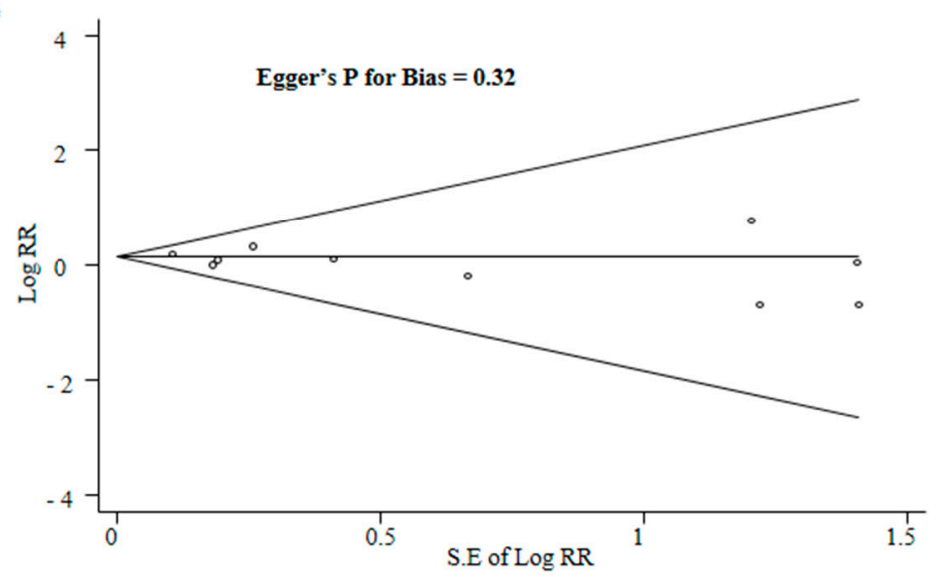

Figure 3. Begg's funnel plots and Egger's test for identifying publication bias of randomized controlled trials. RR, relative risk; CI, confidence interval.

\subsection{Subgroup Meta-Analysis}

The use of calcium supplements was associated with the increased risk of CVD in the subgroup meta-analysis as follows: incidence, healthy postmenopausal women, women, 
participants aged $<65$ years, United States, not specified calcium preparation, duration of calcium supplementation $\geq 5$ years, dosage of calcium supplements $\leq 1000 \mathrm{mg} / \mathrm{d}$ and $1000 \mathrm{mg} / \mathrm{d}$, concurrent use of vitamin D, number of study participants $>1000$, and daily mean dietary calcium intake of 700-1000 mg/d (Table S2). Remarkably, the trials with low risk of bias in at least five items showed a significantly increased risk of CVD in the calcium supplementation group (RR 1.15, 95\% CI 1.06-1.25, $\mathrm{I}^{2}=0.0 \%, n=8$ ), whereas those with low risk of bias in fewer than five items did not show a significant association with the risk of CVD in the calcium supplementation group.

Similarly, calcium supplementation significantly increased the risk of CHD in the subgroup meta-analysis (Table S3). Like the risk of CVD, the trials with low risk of bias in at least five items showed a significantly increased risk of CHD in the calcium supplementation group (RR 1.16, 95\% CI 1.05-1.29, $\mathrm{I}^{2}=0.0 \%, n=8$ ), whereas the only trial with low risk of bias in fewer than five items did not show a significant association with the risk of CHD in the calcium supplementation group (RR 0.65, 95\% CI 0.09-4.56). Additionally, a significant increased risk of CHD in the intervention group was observed in published data (Table S3).

Table S4 shows that, overall, the use of calcium supplements was not associated with the risk of cerebrovascular disease in the subgroup meta-analysis by various factors.

The absolute risk difference for CVD and CHD by using calcium supplements was 8.6 per 1000 persons (0.0086) and 8.8 per 1000 persons (0.0088), respectively.

Figure 3 shows the findings from the leave-one-out sensitivity meta-analysis for each outcome. When Bolland et al.'s study in 2011 was excluded, calcium supplementation was marginally associated with an increased risk of CVD, while all the other leave-one-out sensitivity meta-analyses showed a significantly robust increased risk of CVD (Figure 3A). Additionally, a sensitivity meta-analysis excluding Bolland et al.'s study in 2011 showed that calcium supplementation was not associated with the risk of CHD, while all the other sensitivity meta-analyses did a significantly increased risk of CHD by calcium supplementation (Figure 3B). There was no significant association between calcium supplementation and cerebrovascular disease in any sensitivity meta-analyses (Figure 3C).

\subsection{Differences among the Previous Systematic Reviews and Meta-Analyses and the Current Study}

Table 2 shows the differences in main findings and conclusions among the previous systematic reviews and meta-analyses and the current study. Out of five $[4-6,10,27]$ reviews, three $[4,5,27]$ concluded that calcium supplementation increased or might increase the risk of CVD, similar to our findings. On the contrary, in the remaining two reviews, Lewis et al. [6] concluded that calcium supplementation did not increase the risk of CHD, and 2016 Chung et al. [10] concluded that it had a small risk, which was not considered clinically important, if any; moreover, Chung et al. did not perform a meta-analysis on the association between calcium supplementation and the risk of CVD, but rather performed a qualitative review. These inconsistent findings are thought to be attributable to different selection criteria that were used in each meta-analysis, such as the type of study, study population, and inclusion of unpublished data. Only Lewis et al.'s review, which concluded no increased risk of CVD by calcium supplementation, included open-label trials with no use of placebos or no treatment as a control group. Thus, for example, regarding the risk of myocardial infarction, Bolland et al., Bolland et al., Mao et al., and our research reported similar RRs, ranging from 1.24 to 1.28, whereas Lewis et al. obtained an RR of 1.08. Additionally, Chung et al. included only generally healthy adults as study participants and did not include unpublished data. Thus, they included only four trials, which was too small compared to the other reviews. Chung et al. declared that their study was supported by an unrestricted educational grant from the NOF through Pfizer Consumer Healthcare. 


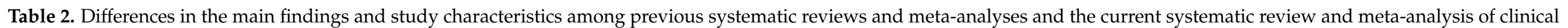
trials on calcium supplementation and the risk of cardiovascular disease.

\begin{tabular}{|c|c|c|c|c|c|c|}
\hline & 2010, Bolland et al. [4] & 2011, Bolland et al. [5] & 2013, Mao et al. [28] & 2015, Lewis et al. [6] & 2016, Chung et al. [10] & $\begin{array}{c}\text { Current } \\
\text { Meta-Analysis }\end{array}$ \\
\hline $\begin{array}{c}\text { Conclusion on Calcium } \\
\text { Supplementation and Risk of CVD }\end{array}$ & Increase & Increase & Might Increase & Not Increase & $\begin{array}{l}\text { Not Associated, Small } \\
\text { Risk and Not } \\
\text { Clinically Important, } \\
\text { if Any }\end{array}$ & Increase \\
\hline \multicolumn{7}{|c|}{ Main Findings: RR $(95 \%$ CI), Number of Included Trials (Reference No.) *, Interpretation in Each Article } \\
\hline Myocardial Infarction (MI) & $\begin{array}{ll}- & 1.27(1.01-1.59) \\
- & 7(16,19,21,22, \\
& 25,26) \\
- & \text { Increased risk }\end{array}$ & $\begin{array}{ll}- & 1.24(1.07-1.45) \\
- & 8(16,19,21,22, \\
& 24,25,26) \\
- & \text { Increased risk }\end{array}$ & $\begin{array}{ll}- & 1.28(0.97-1.68) \\
- & 8(16,19,21,22, \\
& 24,25,26) \\
-\quad & \text { Non- } \\
& \text { significantly } \\
& \text { increased risk }\end{array}$ & $\begin{array}{ll}- & 1.08(0.93-1.25) \\
- & 8(19,21,24,25, \\
& 26,2004 \text { Larsen, } \\
& 2012 \text { Sambrook }) \\
- & \text { No increased } \\
\text { risk }\end{array}$ & n.a. & $\begin{array}{ll}- & 1.25 \\
& (1.07-1.45) \\
-\quad & 9(16,18,19, \\
& 21,22,24,25, \\
& 26) \\
-\quad & \text { Significantly } \\
& \text { increased risk }\end{array}$ \\
\hline Stroke & $\begin{array}{ll}- & 1.12(0.92-1.36) \\
- & 8(1993 \text { Reid, } 16 \\
& 19,20,21,25,26) \\
- & \text { No increased risk }\end{array}$ & $\begin{array}{ll}- & 1.15(1.00-1.32) \\
- & 9(1993 \text { Reid, } 16 \\
& 19,20,21,24,25 \\
& 26) \\
- & \text { Increased risk }\end{array}$ & $\begin{array}{ll}- & 1.14(0.90-1.46) \\
- & \text { Not specified } \\
- & \text { Non- } \\
& \text { significantly } \\
& \text { increased risk }\end{array}$ & n.a. & n.a. & $\begin{array}{ll}- & 1.13 \\
& (0.97-1.31) \\
-\quad & 12(14-18,20- \\
& 22,24-26) \\
-\quad & \text { Non- } \\
& \text { significantly } \\
& \text { increased risk }\end{array}$ \\
\hline $\begin{array}{l}\text { Cardiovascular disease (CVD): coronary } \\
\text { heart disease (CHD) plus stroke }\end{array}$ & $\begin{array}{ll}- & 1.12(0.97-1.30) \\
- & 8(1993 \text { Reid, 16, } \\
\text { 19, 21, 22, 25, 26) } \\
\text { - } & \text { No increased risk } \\
& \text { (composite end } \\
& \text { point of MI, } \\
\text { stroke, and } \\
\text { sudden death) }\end{array}$ & $\begin{array}{ll}- & 1.15(1.03-1.27) \\
- & 10(1993 \text { Reid, 16, } \\
& 19,20,21,22,24, \\
& 25,26) \\
-\quad & \text { Increased risk } \\
& \text { (MI or stroke) }\end{array}$ & $\begin{array}{ll}- & 1.16(0.97-1.40) \\
- & \text { Not specified } \\
- & \text { Non- } \\
& \text { significantly } \\
& \text { increased risk } \\
& \text { (major CV } \\
& \text { events) }\end{array}$ & $\begin{array}{ll}- & 1.02(0.96-1.09) \\
- & 6(19,24,25, \\
& 2004 \text { Larsen, } \\
& 2012 \text { Sambrook }) \\
- & \text { No increased } \\
& \text { risk (CHD) }\end{array}$ & $\begin{array}{ll}\text { - } & \text { No meta-analysis } \\
\text { - } & \text { performed } \\
& 4(2011 \text { Lewis, 24, } \\
& 25,26) \\
- & \text { No statistically } \\
\text { significant } \\
\text { difference }\end{array}$ & $\begin{array}{ll}- & 1.15 \\
& (1.06-1.25) \\
- & 14(14-26) \\
- & \text { Significantly } \\
& \text { increased risk } \\
& \text { (CHD plus } \\
& \text { stroke) }\end{array}$ \\
\hline
\end{tabular}


Table 2. Cont.

\begin{tabular}{|c|c|c|c|c|c|c|c|}
\hline & & 2010, Bolland et al. [4] & 2011, Bolland et al. [5] & 2013, Mao et al. [28] & 2015, Lewis et al. [6] & 2016, Chung et al. [10] & $\begin{array}{c}\text { Current } \\
\text { Meta-Analysis }\end{array}$ \\
\hline \multicolumn{2}{|c|}{$\begin{array}{c}\text { Conclusion on Calcium } \\
\text { Supplementation and Risk of CVD }\end{array}$} & Increase & Increase & Might Increase & Not Increase & $\begin{array}{l}\text { Not Associated, Small } \\
\text { Risk and Not } \\
\text { Clinically Important, } \\
\text { if Any }\end{array}$ & Increase \\
\hline \multicolumn{8}{|c|}{ Main Findings: RR $\left(95 \%\right.$ CI), Number of Included Trials (Reference No.) ${ }^{*}$, Interpretation in Each Article } \\
\hline \multirow{4}{*}{$\begin{array}{l}\text { Selection } \\
\text { Criteria }\end{array}$} & Type of Trials & $\begin{array}{c}\text { Randomized, } \\
\text { double-blind, } \\
\text { placebo-controlled } \\
\text { trials with }>1 \text { year of } \\
\text { trial duration }\end{array}$ & $\begin{array}{c}\text { Randomized, } \\
\text { double-blind, } \\
\text { placebo-controlled } \\
\text { trials }\end{array}$ & $\begin{array}{c}\text { Randomized, } \\
\text { placebo-controlled } \\
\text { trials with at least } \\
\text { one year of follow-up }\end{array}$ & $\begin{array}{c}\text { Randomized } \\
\text { placebo-controlled } \\
\text { trials and open-label } \\
\text { trials }\end{array}$ & $\begin{array}{l}\text { Randomized controlled } \\
\text { trials }\end{array}$ & $\begin{array}{c}\text { Randomized, } \\
\text { double-blind, } \\
\text { placebo-controlled } \\
\text { trials }\end{array}$ \\
\hline & $\begin{array}{c}\text { Inclusion of Trials with } \\
\text { No Use of Placebos }\end{array}$ & No & No & No & Yes & No & No \\
\hline & Study Participants & $\begin{array}{c}\text { Participants aged }>40 \\
\text { years }\end{array}$ & $\begin{array}{c}\text { Participants aged }>40 \\
\text { years }\end{array}$ & Not described & $\begin{array}{l}\text { A mean cohort age } \\
>50 \text { years }\end{array}$ & $\begin{array}{l}\text { Generally healthy } \\
\text { adults }\end{array}$ & Adults \\
\hline & $\begin{array}{c}\text { Inclusion of } \\
\text { Unpublished Data }\end{array}$ & Yes & Yes & Yes & Yes & No & Yes \\
\hline \multicolumn{2}{|c|}{ Subgroup meta-analysis } & $\begin{array}{l}\text { Type of endpoints (MI, } \\
\text { stroke, and death) }\end{array}$ & $\begin{array}{c}\text { Type of endpoints (MI, } \\
\text { stroke, and MI + } \\
\text { stroke) }\end{array}$ & $\begin{array}{l}\text { Type of endpoints } \\
\text { (major cardiovascular } \\
\text { events, MI, stroke) } \\
\text { and sex }\end{array}$ & $\begin{array}{l}\text { Type of endpoints } \\
\text { (MI, angina pectoris } \\
\text { and acute coronary } \\
\text { syndrome, chronic } \\
\text { coronary heart } \\
\text { disease, and all-cause } \\
\text { mortality) }\end{array}$ & n.a. & $\begin{array}{l}\text { Type of endpoints } \\
\text { (MI, angina } \\
\text { pectoris, coronary } \\
\text { revascularization, } \\
\text { stroke, coronary } \\
\text { heart disease, } \\
\text { CVD), low risk of } \\
\text { bias, population, } \\
\text { age, gender, region, } \\
\text { dosage, duration of } \\
\text { supplementation, } \\
\text { and data source } \\
\text { (published or } \\
\text { unpublished) }\end{array}$ \\
\hline
\end{tabular}


Table 2. Cont.

2010, Bolland et al. [4]

2011, Bolland et al. [5]

2013, Mao et al. [28]

2015, Lewis et al. [6]

2016, Chung et al. [10]

Not Associated, Small

Risk and Not

Clinically Important

if Any

Not Increase

Supplementation and Risk of CVD

Increase

Increase

Might Increase

Main Findings: RR (95\% CI), Number of Included Trials (Reference No.) ${ }^{*}$, Interpretation in Each Article

\begin{tabular}{|c|c|c|}
\hline \multirow{5}{*}{ Funding Source } & The Health Research & The Health Research \\
\hline & Council of New & Council of New \\
\hline & Zealand and the & Zealand and the \\
\hline & University of & University of \\
\hline & $\begin{array}{l}\text { Auckland School of } \\
\text { Medicine Foundation }\end{array}$ & $\begin{array}{l}\text { Auckland School of } \\
\text { Medicine Foundation }\end{array}$ \\
\hline
\end{tabular}

National "Eleven

new drugs

creation" special

science and

technology major,

a major national

science and
Current

Meta-Analysis

Increase

technology

$$
\text { etc. }
$$

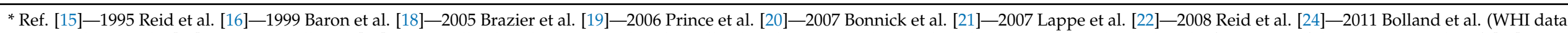

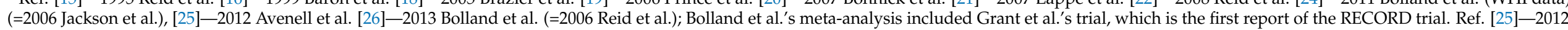

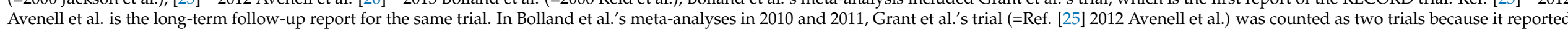

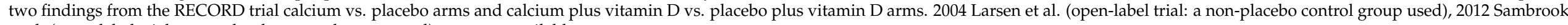
et al. (open-label trial a non-placebo control group used); n.a., not available. 


\section{Discussion}

We found that calcium supplements were significantly associated with an increased risk of CVD and CHD in a meta-analysis of 28,935 participants from 13 double-blind, placebo-controlled RCTs, specifically in healthy postmenopausal women. In the subgroup meta-analysis, dietary calcium intake of 700-1000 mg per day or supplementary calcium intake of $1000 \mathrm{mg}$ per day significantly increased the risk of CVD and CHD. There was no significant association between the use of calcium supplements and the risk of cerebrovascular disease.

There are several possible biological mechanisms that could explain the increased risk of CVD with the use of calcium supplements observed in our meta-analysis of doubleblind, placebo-controlled RCTs. Calcium supplementation could elevate circulating calcium concentrations and, consequently, lead to the development of CVD. First, clinical trials reported that oral calcium supplementation raised serum calcium concentrations [29], which remained elevated after long-term administration of calcium supplements [30]. Furthermore, a randomized controlled trial found that serum calcium levels increased higher after skim milk powder enriched with calcium carbonate supplements than skim powder enriched with milk calcium [31], which might indicate that the intakes of calcium supplements such as calcium carbonate have larger biological effects than dietary calcium intakes such as dairy products. For instance, two meta-analyses of prospective cohort studies $[32,33]$ concluded that dietary calcium intake was not associated with the risk of CVD by comparing the highest and lowest intake levels, while one of them [30] also reported a non-linear association between dietary calcium intake and the risk of CVD mortality. Second, elevated calcium concentrations after calcium supplementation may increase vascular calcification, which is considered as an established factor for CVD. An in vitro study using human vascular smooth muscle cells reported that elevated calcium or phosphate-induced vascular calcification occurred independently and synergistically [34]. Vascular calcification is considered to be a regulated process involving many inhibitors of crystal formation, especially pyrophosphate [35]. Additionally, it was reported that downregulation of the calcium-sensing receptor due to increased concentrations of calcium results in increased mineralization of the vascular smooth muscle cell cultures, which is another mechanism of vascular calcification by serum calcium [36]. For instance, a retrospective study found that elevated serum levels of calcium, even within the normal range, was significantly associated with the presence of calcified coronary atherosclerotic plaque assessed by cardiac computed tomography angiography [37]. Third, another possible mechanism might be blood coagulation. Free ionized calcium is an essential cofactor for several interactions in the coagulation cascade, and it was reported that whole blood clotting time was prolonged in rats with ionized hypocalcemia induced by rapid intravenous citrate infusion [38]. Conversely, Bristow et al. reported that the administration of calcium supplements increased the coagulation index by about $20 \%$ in a randomized placebo-controlled trial of postmenopausal women [39]. Lastly, elevated serum calcium levels are associated with the increased risk of CVD. Reid et al.'s systematic review of observational studies demonstrated that there was a positive association between serum calcium and CVD [40]. Additionally, Larsson et al.'s Mendelian randomization study found that a genetic predisposition to higher serum calcium levels increased the risk of coronary artery disease and myocardial infarction [41].

Our findings are consistent with those from the previous two meta-analyses of RDPCTs by Bolland and colleagues [4,5], while the other two meta-analyses of randomized trials by Lewis et al. [6] and Chung et al. [10] reported no association between calcium supplementation and the risk of CVD unlike our findings. Mao et al.'s meta-analysis also reported that calcium supplementation alone might increase the risk of CVD, although the difference was not statistically significant [28]. As mentioned in the results section, these inconsistent findings are thought to be attributable to different selection criteria, such as type of study, study population, and inclusion of unpublished data. 
In the subgroup meta-analysis by mean daily dietary calcium intake, dietary calcium intake of 700-1000 mg/d significantly increased the risk of CVD, while there was no significant association between calcium intakes of lower or higher than 700-1000 mg/d intake and the risk of CVD. However, the number of the included trials for those ranges of calcium intakes was too small to draw a conclusion. Additionally, calcium supplements of $1000 \mathrm{mg} / \mathrm{d}$ significantly increased the risk of CVD and CHD. Even though calcium supplements with a concentration lower or higher than $1000 \mathrm{mg} / \mathrm{d}$ showed a non-significant association with the risk of CVD and CHD, there was a trend of increased risks of CVD. Further trials are warranted to confirm this finding.

Our study has several limitations. First, most of the double-blind, placebo-controlled RCTs included in our analysis were not designed specifically to investigate the effect of calcium supplements on the risk of CVD as the primary endpoint. In general, findings in the secondary endpoint might be due to chance because the design of the trial is not specifically powered to assess it. However, because conducting a double-blind, placebocontrolled RCT by using only the risk of CVD as the primary endpoint is unethical, further double-blind, placebo-controlled RCTs should consider both the incidence of osteoporosis or fractures and CVD as the primary endpoint, not just as the secondary endpoint. Second, we used unpublished data provided by authors for $5[14,15,17,20,21]$ out of 13 doubleblind, placebo-controlled RCTs, which were obtained from Bolland et al.'s meta-analysis article [4]. This might be associated with selection bias. However, when we performed a subgroup meta-analysis excluding those five trials, a significant increased risk of CVD and CHD in the calcium supplementation group was still observed. Third, the majority of study participants in the RDBCTs $(92.8 \%)$ were postmenopausal women with a mean age of 66.3 years. Thus, our findings should be applied to only postmenopausal women. Fourth, the current meta-analysis was not registered at PROSPERO. Last, in the leaveone-out sensitivity meta-analysis for each outcome, when Bolland et al.'s study in 2011 was excluded, calcium supplementation turned out to be marginally associated with an increased risk of CVD and non-significantly associated with an increased risk of CHD. This is attributable to a large sample size of Bolland et al.'s study in 2011, which used the data from the Women's Health Initiative study involving 16,718 study participants. Thus, further large, double-blind, placebo-controlled RCTs are required to confirm our findings.

In spite of these limitations, our findings have a significant implication. It has been reported that up to $50 \%$ of older women take calcium supplements in Western countries [27]. However, a recent meta-analysis [42] of 33 RCTs reported that the use of calcium or vitamin D supplementation was not associated with a lower risk of fractures in older adults, and our study found that the use of calcium supplements might have potential CVD risks. Therefore, in terms of 'precautionary principle' as well as evidence-based medicine, supplementary calcium intakes should be discouraged.

\section{Conclusions}

The current meta-analysis of double-blind, placebo-controlled RCT showed that the use of calcium supplements was significantly associated with the increased risk of CVD and CHD by $15 \%$, specifically in postmenopausal women. Our findings should be explicitly confirmed by conducting further RDBCTs with CVD outcome measures as well as the incidence of osteoporosis or fractures as the primary endpoints.

Supplementary Materials: The following are available online at https:/ / www.mdpi.com/2072-6 643/13/2/368/s1, Table S1: Summary of risk of bias assessment for randomized, double-blind, placebo-controlled trials, Table S2: Use of calcium supplements and risk of cardiovascular disease (CVD) in the subgroup meta-analysis of randomized, double-blind, placebo-controlled trials, Table S3: Use of calcium supplements and risk of coronary heart disease (CHD) in the subgroup metaanalysis of randomized, double-blind, placebo-controlled trials, Table S4: Use of calcium supplements and risk of cerebrovascular disease in the subgroup meta-analysis of randomized, double-blind, placebo-controlled trials $(n=13)$. 
Author Contributions: S.-K.M. has full access to all of the data in the study and takes responsibility for the integrity of the data and the accuracy of the data analysis. Conceptualization, S.-K.M.; literature search and assessment of study eligibility, H.-B.K. and Y.-J.L.; assessment of methodological quality, H.-B.K., S.-K.M., S.-W.O., and Y.-J.C.; statistical analysis and writing-original draft preparation, H.-B.K. and S.-K.M.; writing-review and editing, S.-K.M. and H.-B.K. All authors have read and agreed to the published version of the manuscript.

Funding: This research received no external funding.

Institutional Review Board Statement: Not applicable.

Informed Consent Statement: Not applicable.

Data Availability Statement: The authors used published data from the individual studies and declare that the data supporting the findings of this study are available within the article.

Conflicts of Interest: The authors declare no conflict of interest.

\section{References}

1. Cosman, F.; de Beur, S.J.; LeBoff, M.S. Clinician's Guide to Prevention and Treat-ment of Osteoporosis. Osteoporos. Int. 2014, 25, 2359-2381. [CrossRef] [PubMed]

2. Camacho, P.M.; Petak, S.M.; Binkley, N. American association of clinical endocrinologists and american college of endocrinology clinical practice guidelines for the diagnosis and treatment of post-menopausal osteoporosis. Endocr. Pract. 2016, 22 (Suppl. 4), 1-42. [CrossRef] [PubMed]

3. Compston, J.E.; The National Osteoporosis Guideline Group (NOGG); Cooper, A.; Cooper, C.; Gittoes, N.; Gregson, C.L.; Harvey, N.C.; Hope, S.; Kanis, J.A.; McCloskey, E. UK clinical guideline for the prevention and treatment of osteoporosis. Arch. Osteoporos. 2017, 12, 1-24. [CrossRef] [PubMed]

4. Bolland, M.J.; Avenell, A.; Baron, J.A. Effect of calcium supplements on risk of myo-cardial infarction and cardiovascular events: Meta-analysis. BMJ 2010, 341, c3691. [CrossRef] [PubMed]

5. Bolland, M.J.; Grey, A.; Avenell, A. Calcium supplements with or without vitamin D and risk of cardiovascular events: Reanalysis of the Women's Health Initiative lim-ited access dataset and meta-analysis. BMJ 2011, 342, d2040. [CrossRef]

6. Lewis, J.R.; Radavelli-Bagatini, S.; Rejnmark, L. The effects of calcium supplemen-tation on verified coronary heart disease hospitalization and death in postmenopausal women: A collaborative meta-analysis of randomized controlled trials. J. Bone Miner. Res. 2015, 30, 165-175. [CrossRef]

7. Nordin, B.E.; Lewis, J.R.; Daly, R.M. The calcium scare-What would Austin Brad-ford Hill have thought? Osteoporos. Int. 2011, 22, 3073-3077. [CrossRef]

8. Bolland, M.J.; Grey, A.; Reid, I.R. Re: The calcium scare: What would Austin Bradford Hill have thought? Osteoporos. Int. 2011, 22, 3079-3080. [CrossRef]

9. Bolland, M.J.; Grey, A.; Avenell, A.; Reid, I.R. Calcium supplements increase risk of myo-cardial infarction. J. Bone. Miner. Res. 2015, 30, 389-390. [CrossRef]

10. Chung, M.; Tang, A.M.; Fu, Z. Calcium Intake and cardiovascular Disease Risk: An Updated Systematic Review and Meta-analysis. Ann. Intern. Med. 2016, 165, 856-866. [CrossRef]

11. Kopecky, S.; Bauer, D.C.; Gulati, M.; Nieves, J.W.; Singer, A.J.; Toth, P.P.; Underberg, J.; Wallace, T.; Weaver, C.M. Lack of Evidence Linking Calcium With or Without Vitamin D Supplementation to Cardiovascular Disease in Generally Healthy Adults: A Clinical Guideline from the National Osteoporosis Foundation and the American Society for Preventive Cardiology. Ann. Intern. Med. 2016, 165, 867. [CrossRef] [PubMed]

12. Higgins, J.P.T.; Savović, J.; Page, M.J.; Elbers, R.G.; Sterne, J.A.C. Chapter 8: Assessing risk of bias in a randomized trial. In: Higgins JPT, Thomas J, Chandler J, Cumpston M, Li T, Page MJ, Welch VA (editors). Cochrane Handbook for Systematic Reviews of Interventions version 6.1 (updated September 2020). Cochrane, 2020. Available online: www.training.cochrane.org/handbook (accessed on 1 November 2020).

13. Higgins, J.P.T.; Thompson, S.G. Quantifying heterogeneity in a meta-analysis. Stat. Med. 2002, 21, 1539-1558. [CrossRef] [PubMed]

14. Dawson-Hughes, B.; Dallal, G.E.; Krall, E.A. A controlled trial of the effect of cal-cium supplementation on bone density in postmenopausal women. N. Engl. J. Med. 1990, 323, 878-883. [CrossRef] [PubMed]

15. Reid, I.R.; Ames, R.W.; Evans, M.C.; Gamble, G.D.; Sharpe, S.J. Long-term effects of calcium supplementation on bone loss and fractures in postmenopausal women: A randomized controlled trial. Am. J. Med. 1995, 98, 331-335. [CrossRef]

16. Baron, J.; Beach, M.; Mandel, J.; Van Stolk, R.; Haile, R.; Sandler, R.; Rothstein, R.; Summers, R.; Snover, D.; Beck, G.; et al. Calcium Supplements for the Prevention of Colorectal Adenomas. N. Engl. J. Med. 1999, 340, 101-107. [CrossRef]

17. Bonithon-Kopp, C.; Kronborg, O.; Giacosa, A.; Räth, U.; Faivre, J. Calcium and fibre supplementation in prevention of colorectal adenoma recurrence: A randomised intervention trial. Lancet 2000, 356, 1300-1306. [CrossRef] 
18. Brazier, M.; Grados, F.; Kamel, S. Clinical and laboratory safety of one year's use of a combination calcium + vitamin D tablet in ambulatory elderly women with vita-min D insufficiency: Results of a multicenter, randomized, double-blind, place-bo-controlled study. Clin. Ther. 2005, 27, 1885-1893. [CrossRef]

19. Prince, R.L.; Devine, A.; Dhaliwal, S.S. Effects of calcium supplementation on clin-ical fracture and bone structure: Results of a 5-year, double-blind, placebo-controlled trial in elderly women. Arch. Intern. Med. 2006, 166, 869-875. [CrossRef]

20. Bonnick, S.L.; Broy, S.; Kaiser, F.; Teutsch, C.; Rosenberg, E.; Delucca, P.; Melton, M. Treatment with alendronate plus calcium, alendronate alone, or calcium alone for postmenopausal low bone mineral density. Curr. Med. Res. Opin. 2007, 23, 1341-1349. [CrossRef] [PubMed]

21. Lappe, J.M.; Travers-Gustafson, D.; Davies, K.M. Vitamin D and calcium supple-mentation reduces cancer risk: Results of a randomized trial. Am. J. Clin. Nutr. 2007, 85, 1586-1591. [CrossRef]

22. Reid, I.R.; Ames, R.; Mason, B. Randomized controlled trial of calcium supple-mentation in healthy, non-osteoporotic, older men. Arch. Intern. Med. 2008, 168, 2276-2282. [CrossRef]

23. Chailurkit, L.O.; Saetung, S.; Thakkinstian, A. Discrepant influence of vitamin D status on parathyroid hormone and bone mass after two years of calcium supplemen-tation. Clin. Endocrinol. 2010, 73, 167-172.

24. Bolland, M.J.; Grey, A.; Gamble, G.D.; Reid, I.R. Calcium and vitamin D supplements and health outcomes: A reanalysis of the Women's Health Initiative (WHI) limited-access data set. Am. J. Clin. Nutr. 2011, 94, 1144-1149. [CrossRef] [PubMed]

25. Avenell, A.; MacLennan, G.; Jenkinson, D.J.; McPherson, G.; McDonald, A.; Pant, P.R.; Grant, A.M.; Campbell, M.K.; Anderson, F.H.; Cooper, C.; et al. Long-Term Follow-Up for Mortality and Cancer in a Randomized Placebo-Controlled Trial of Vitamin D3and/or Calcium (RECORD Trial). J. Clin. Endocrinol. Metab. 2012, 97, 614-622. [CrossRef]

26. Bolland, M.J.; Barber, A.; Doughty, R.N.; Grey, A.; Gamble, G.; Reid, I.R. Differences between self-reported and verified adverse cardiovascular events in a randomised clinical trial. BMJ Open 2013, 3, e002334. [CrossRef]

27. Bolland, M.J.; Leung, W.; Tai, V.; Bastin, S.; Gamble, G.D.; Grey, A.; Reid, I.R. Calcium intake and risk of fracture: Systematic review. BMJ 2015, 351, h4580. [CrossRef] [PubMed]

28. Mao, P.-J.; Zhang, C.; Tang, L.; Xian, Y.-Q.; Li, Y.-S.; Wang, W.-D.; Zhu, X.-H.; Qiu, H.-L.; He, J.; Zhou, Y.-H. Effect of calcium or vitamin D supplementation on vascular outcomes: A meta-analysis of randomized controlled trials. Int. J. Cardiol. 2013, 169, 106-111. [CrossRef] [PubMed]

29. Guillemant, J.; Guillemant, S. Comparison of the suppressive effect of two doses (500 mg vs. $1500 \mathrm{mg}$ ) of oral calcium on parathyroid hormone secretion and on urinary cyclic AMP. Calcif. Tissue Int. 1993, 53, 304-306. [CrossRef]

30. Bristow, S.M.; Gamble, G.D.; Stewart, A.; Horne, L.; House, M.E.; Aati, O.; Mihov, B.; Horne, A.M.; Reid, I.R. Acute and 3-month effects of microcrystalline hydroxyapatite, calcium citrate and calcium carbonate on serum calcium and markers of bone turnover: A randomised controlled trial in postmenopausal women. Br. J. Nutr. 2014, 112, 1611-1620. [CrossRef]

31. Green, J.H.; Booth, C.; Bunning, R. Postprandial metabolic responses to milk enriched with milk calcium are different from responses to milk enriched with calcium car-bonate. Asia Pac. J. Clin. Nutr. 2003, 12, 109-119.

32. Wang, L.; Manson, J.E.; Sesso, H.D. Calcium Intake and Risk of Cardiovascular Disease. Am. J. Cardiovasc. Drugs 2012, 12, 105-116. [CrossRef] [PubMed]

33. Wang, X.; Chen, H.; Ouyang, Y.; Liu, J.; Zhao, G.; Bao, W.; Yan, M. Dietary calcium intake and mortality risk from cardiovascular disease and all causes: A meta-analysis of prospective cohort studies. BMC Med. 2014, 12, 158. [CrossRef] [PubMed]

34. Reynolds, J.L.; Joannides, A.J.; Skepper, J.N. Human vascular smooth muscle cells undergo vesicle-mediated calcification in response to changes in extracellular calcium and phosphate concentrations: A potential mechanism for accelerated vascular calci-fication in ESRD. J. Am. Soc. Nephrol. 2004, 15, 2857-2867. [CrossRef] [PubMed]

35. Reid, I.R.; Bolland, M.J.; Grey, A. Does calcium supplementation increase cardiovascular risk? Clin. Endocrinol. 2010, 73, 689-695. [CrossRef] [PubMed]

36. Alam, M.-U.; Kirton, J.P.; Wilkinson, F.L.; Towers, E.; Sinha, S.; Rouhi, M.; Vizard, T.N.; Sage, A.P.; Martin, D.; Ward, D.T.; et al. Calcification is associated with loss of functional calcium-sensing receptor in vascular smooth muscle cells. Cardiovasc. Res. 2008, 81, 260-268. [CrossRef] [PubMed]

37. Shin, S.; Kim, K.J.; Chang, H.J. Impact of serum calcium and phosphate on coro-nary atherosclerosis detected by cardiac computed tomography. Eur. Heart J. 2012, 33, 2873-2881. [CrossRef]

38. Fukuda, T.; Nakashima, Y.; Harada, M.; Toyoshima, S.; Koshitani, O.; Kawaguchi, Y.; Nakayama, M. Effect of whole blood clotting time in rats with ionized hypocalcemia induced by rapid intravenous citrate infusion. J. Toxicol. Sci. 2006, 31, 229-234. [CrossRef]

39. Bristow, S.M.; Gamble, G.; Stewart, A.; Horne, A.; Reid, I.R. Acute effects of calcium supplements on blood pressure and blood coagulation: Secondary analysis of a randomised controlled trial in post-menopausal women. Br. J. Nutr. 2015, 114, 1868-1874. [CrossRef]

40. Reid, I.R.; Gamble, G.D.; Bolland, M.J. Circulating calcium concentrations, vascular dis-ease and mortality: A systematic review. J. Intern. Med. 2016, 279, 524-540. [CrossRef]

41. Larsson, S.C.; Burgess, S.; Michaëlsson, K. Association of Genetic Variants Related to Serum Calcium Levels With Coronary Artery Disease and Myocardial Infarction. JAMA 2017, 318, 371-380. [CrossRef]

42. Zhao, J.G.; Zeng, X.T.; Wang, J.; Liu, L. Association Between Calcium or Vitamin D Supplementation and Fracture Incidence in Community-Dwelling Older Adults A Sys-tematic Review and Meta-analysis. JAMA 2017, 318, 2466-2482. [CrossRef] [PubMed] 